\title{
Non-Symmetric Flow over a Stretching/Shrinking Surface with Mass Transfer
}

\author{
Yian Yian Lok ${ }^{a}$, John H. Merkin ${ }^{b}$ and Ioan Pop ${ }^{c}$ \\ ${ }^{a}$ Mathematics Section, School of Distance Education, Universiti Sains \\ Malaysia \\ 11800 USM, Pulau Pinang, Malaysia \\ ${ }^{b}$ Department of Applied Mathematics, University of Leeds \\ Leeds, LS2 9JT, UK \\ ${ }^{c}$ Department of Mathematics, Babeş-Bolyai University \\ 400084 Cluj-Napoca, Romania \\ E-mail(corresp.): amtjhm@maths.leeds.ac.uk \\ E-mail: lokyy@usm.my \\ E-mail: popm.ioan@yahoo.co.uk
}

Received March 18, 2019; revised October 10, 2019; accepted October 7, 2019

\begin{abstract}
The non-symmetric flow over a stretching/shrinking surface in an otherwise quiescent fluid is considered under the assumption that the surface can stretch or shrink in one direction and stretch in a direction perpendicular to this. The problem is reduced to similarity form, being described by two dimensionless parameters, $\gamma$ the relative stretching/shrinking rate and $S$ characterizing the fluid transfer through the boundary. Numerical solutions are obtained for representative values of $\gamma$ and $S$, a feature of which are the existence of critical values $\gamma_{c}$ of $\gamma$ dependent on $S$, these being determined numerically. Asymptotic forms for large $\gamma$ and $S$, for both fluid withdrawal, $S>0$ and injection $S<0$ are obtained and compared with the corresponding numerical results.
\end{abstract}

Keywords: non-symmetric flow, permeable surface, multiple solutions, asymptotic solutions.

AMS Subject Classification: 34B15; 65L07; 76M25; 76M45.

Copyright (c) 2019 The Author(s). Published by VGTU Press

This is an Open Access article distributed under the terms of the Creative Commons Attribution License (http://creativecommons.org/licenses/by/4.0/), which permits unrestricted use, distribution, and reproduction in any medium, provided the original author and source are credited. 


\section{Introduction}

Over recent years the analysis of the boundary-layer flows of viscous fluids resulting from continuously moving or stretching/shrinking surfaces has many important applications in both engineering processes and the polymer industry. Examples of such technological processes concerning polymers include the cooling of continuous strips or filaments, glass blowing, the continuous stretching of plastic films and artificial fibres, the continuous casting of metals, the spinning of fibres, hot rolling, wire drawing and paper production, see Fisher [11] for example. A viscous fluid subject to a stretching surface was analyzed first by Crane [8] where an exact and closed form similarity solution was obtained. Gupta and Gupta [13] discussed the heat and mass transfer due to a permeable stretching sheet. They also presented the analysis for both the suction and blowing cases. Banks [4] described a class of similarity solutions depending upon a parameter for the boundary-layer equations caused by a stretching wall. The existence and uniqueness of stretching flows is discussed by McLeod and Rajagopal [20].

Chen and Char [6] studied the heat transfer characteristics on a stretching sheet with a variable surface temperature in the case of suction or blowing. Ali [2] considered the thermal boundary layer on a stretching surface using powerlaw velocity and temperature distributions. More recently, Crane's original problem [8] has been extended to treat the various aspects of the flow and heat transfer characteristics with linear or power-law surface velocities by several authors, in particular by Magyari and Keller [18], Liao and Pop [16], Liao [15], Ishak et al. [14], Vajravelu [27], Cortell [7], Bataller [5], Sajid et al. [25] and literature therein. Wang [29] has reviewed the similarity solutions with an assumption that the stretching surface is large such that edge effects are negligible.

The three-dimensional flow due to the bi-axial stretching of a flat surface, including an axisymmetric stretching surface, was studied by Wang [28] in a quiescent fluid, and by Wang [30] in a fluid with uniform outer flow. These problems lead to the exact solutions of the Navier-Stokes equations. Ariel [3] has demonstrated that highly efficient and very accurate solutions in terms of series of exponentially decaying functions can be obtained by using the Ackyord's method in solving the generalized three-dimensional flow caused by a bi-axial stretching sheet in a quiescent fluid. More recently, Magyari and Weidman [19] investigated the problem of three-dimensional flow induced by moving boundaries where the surface can be moved along and/or transverse to the direction of the stretching at uniform velocities in either quiescent fluids or in fluid with an external uniform shear.

Miklavčič and Wang [24] have investigated the two-dimensional flow towards a shrinking sheet with constant velocity in a viscous fluid and obtained the exact solutions of the full Navier-Stokes equations. It was also shown that mass suction is required to realize the shrinking sheet flow. Fang [9] extended the problem originally treated by Miklavčič and Wang [24] to the shrinking sheet with a power-law velocity distribution. Multiple solutions are obtained and velocity overshoots are observed near the wall and near the boundary 
layer edge for certain solutions branches. The flow induced by an unsteady shrinking sheet with mass suction has been investigated by Fang et al. [10]. Multiple solutions are also obtained for a certain range of mass suction and the unsteadiness parameters. The shrinking sheet flow, as discussed by Goldstein [12], is essentially a backward boundary-layer flow which shows different physical phenomena from the stretching sheet flow.

The aim of the present paper is to extend the work of Wang $[28,30]$ and Ariel [3] to the case of a three-dimensional stretching/shrinking sheet where the fluid is at rest far from the sheet. In the present case, the surface is stretched in one direction but can be stretched or shrunk in a direction perpendicular to the stretching surface. This type of problem is commonly found in the manufacturing of polymer films. During the casting process, the plastic film is extruded continuously (stretching in one direction) while the edge effect of the film such as neck-in (shrink in the perpendicular direction) is naturally formed. We believe that our results are new and additional to those presented previously, which can be used with great confidence by all those who are interested with the stretching/shrinking sheet. We start by considering the equations that describe our model.

\section{Equations}

We consider the steady non-symmetric flow over a permeable stretching/shrinking surface placed in a viscous fluid at rest well away from the surface. We assume a surface velocity of the form

$$
u_{w}=A x, v_{w}=B y, w_{w}=-W_{0},
$$

where, as shown in Figure 1, $x, y$ and $z$ are Cartesian coordinates with $z$ normal to the surface and $x$ and $y$ are measured in the plane $z=0 . u, v$ and $w$ are respectively the velocity components in the $x, y$ and $z$ directions and $A$ and $B$ are constants with $A>0$.

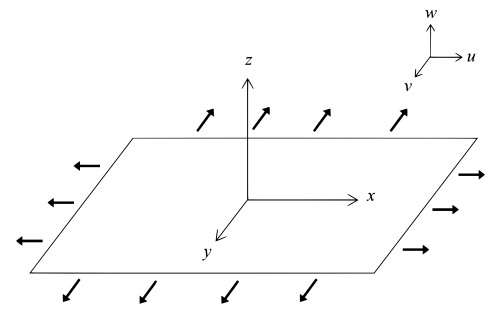

a)

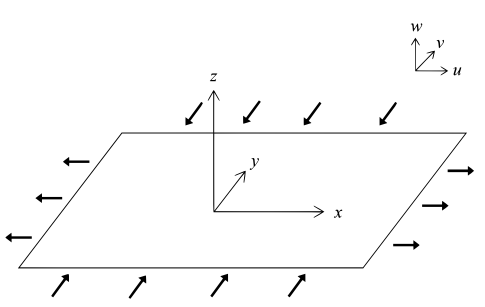

b)

Figure 1. Physical model and co-ordinate system: a) stretching surface, b) shrinking surface.

The basic equations for this problem are, see Wang [30], Magyari and Wei- 
dman [19], Miklavčič and Wang [24],

$$
\begin{aligned}
& \frac{\partial u}{\partial x}+\frac{\partial v}{\partial y}+\frac{\partial w}{\partial z}=0 \\
& u \frac{\partial u}{\partial x}+v \frac{\partial u}{\partial y}+w \frac{\partial u}{\partial z}=-\frac{1}{\rho} \frac{\partial p}{\partial x}+\nu\left(\frac{\partial^{2} u}{\partial x^{2}}+\frac{\partial^{2} u}{\partial y^{2}}+\frac{\partial^{2} u}{\partial z^{2}}\right), \\
& u \frac{\partial v}{\partial x}+v \frac{\partial v}{\partial y}+w \frac{\partial v}{\partial z}=-\frac{1}{\rho} \frac{\partial p}{\partial y}+\nu\left(\frac{\partial^{2} v}{\partial x^{2}}+\frac{\partial^{2} v}{\partial y^{2}}+\frac{\partial^{2} v}{\partial z^{2}}\right), \\
& u \frac{\partial w}{\partial x}+v \frac{\partial w}{\partial y}+w \frac{\partial w}{\partial z}=-\frac{1}{\rho} \frac{\partial p}{\partial z}+\nu\left(\frac{\partial^{2} w}{\partial x^{2}}+\frac{\partial^{2} w}{\partial y^{2}}+\frac{\partial^{2} w}{\partial z^{2}}\right),
\end{aligned}
$$

subject to the boundary conditions

$$
u=u_{w}, v=v_{w}, w=w_{w} \text {, on } z=0, u, v \rightarrow 0 \text { as } z \rightarrow \infty \text {. }
$$

We look for a similarity solution by putting

$$
\begin{aligned}
& u=A x f^{\prime}(\eta), \quad v=B y g^{\prime}(\eta), \\
& w=-(A f(\eta)+B g(\eta)) \sqrt{\nu / A}, \quad \eta=\sqrt{A / \nu} z .
\end{aligned}
$$

This gives

$$
\begin{aligned}
& f^{\prime \prime \prime}+f f^{\prime \prime}-f^{\prime 2}+\gamma g f^{\prime \prime}=0, \\
& g^{\prime \prime \prime}+\gamma\left(g g^{\prime \prime}-g^{\prime 2}\right)+f g^{\prime \prime}=0
\end{aligned}
$$

where $\gamma=B / A$ and subject to the boundary conditions

$$
f^{\prime}(0)=1, g^{\prime}(0)=1, f^{\prime} \rightarrow 0, g^{\prime} \rightarrow 0 \text { as } \eta \rightarrow \infty,
$$

where primes denote differentiation with respect to $\eta$. This present case corresponds to stretching in the $x$-direction and to stretching in the $y$-direction when $\gamma>0$ or shrinking in the $y$-direction when $\gamma<0$.

For an impermeable surface, $W_{0}=0$, we have $f(0)=g(0)=0$. However, for a permeable surface, $W_{0} \neq 0$, the boundary condition is, from $(2.2)$ and $(2.3)$

$$
f(0)+\gamma g(0)=S, \quad \text { where } \quad S=\frac{W_{0}}{\sqrt{\nu A}} .
$$

Here we take, exploiting the symmetry inherent in the basic problem,

$$
f(0)=S, \quad g(0)=0
$$

noting that (2.8) satisfies condition (2.7).

We note that, for $\gamma=1, g=f-S$ giving $f^{\prime \prime}(0)=g^{\prime \prime}(0)$. When $\gamma=0$, equation (2.4) can be solved to obtain

$$
f=c-\frac{1}{c} e^{-c \eta}, \text { where } c=\frac{S+\sqrt{S^{2}+4}}{2}
$$


Table 1. Comparison with previous results for $\gamma=1.0$ and $S=0$.

\begin{tabular}{cccc}
\hline & Present & Wang [30] & Ariel [3] \\
\hline$f^{\prime \prime}(0)$ & -1.173721 & -1.1737 & -1.17372074 \\
$g^{\prime \prime}(0)$ & -1.173721 & -1.1737 & -1.17372074 \\
$f(\infty)$ & 0.751497 & 0.7515 & - \\
$g(\infty)$ & 0.751497 & 0.7515 & - \\
\hline
\end{tabular}

giving

$$
f^{\prime \prime}(0)=-\frac{S+\sqrt{S^{2}+4}}{2}
$$

Equation (2.5) then gives

$$
g^{\prime}=A \exp \left(-\frac{e^{-c \eta}}{c^{2}}\right)+B
$$

for new constants $A$ and $B$. The boundary conditions give

$$
A=-B=-\frac{1}{1-e^{-1 / c^{2}}} \quad \text { giving } \quad g^{\prime \prime}(0)=-\frac{1}{c\left(e^{1 / c^{2}}-1\right)} .
$$

When $S=0$, expressions (2.9)-(2.10) give

$$
f^{\prime \prime}(0)=-1 \text { and } g^{\prime \prime}(0)=-1 /(e-1) \simeq-0.58198 .
$$

We start by considering an impermeable surface, $S=0$.

\section{Impermeable surface, $S=0$}

The boundary-value problem given by equations (2.4)-(2.6) was solved using bvp $4 c$ in MATLAB [26] and D02AGF in the NAG library [1]. In Table 1 we compare our results with those from previous studies, showing excellent agreement and hence giving confidence in the accuracy of our numerical integrations.

In Figure 2 we plot $f^{\prime \prime}(0)$ and $g^{\prime \prime}(0)$ against $\gamma$ for positive values of $\gamma$. We see that the curves for both $f^{\prime \prime}(0)$ and $g^{\prime \prime}(0)$ start at $\gamma=0$ with the values given above, remain negative, cross when $\gamma=1$ and decrease to large values as $\gamma$ is increased.

In Figure 3 we again plot $f^{\prime \prime}(0)$ and $g^{\prime \prime}(0)$ but now for negative values of $\gamma$. Here we see that there is a critical point at $\gamma=\gamma_{c} \simeq-0.2514$ with $f_{c}^{\prime \prime}(0) \simeq-0.9344, g_{c}^{\prime \prime}(0) \simeq-0.1177$. On the upper solution branch, the values of $f^{\prime \prime}(0)$ decrease towards -1 and those of $g^{\prime \prime}(0)$ increase to large positive values as $\gamma \rightarrow 0$ from below.

As $\gamma$ becomes small on the upper branch solutions a double-layer structure develops, as can be seen in Figure 4 where we plot $f, f^{\prime}$ and $g^{\prime}$ against $\eta$ for $\gamma=-0.01253$. The region where $f$ and $f^{\prime}$ vary remains relatively small 


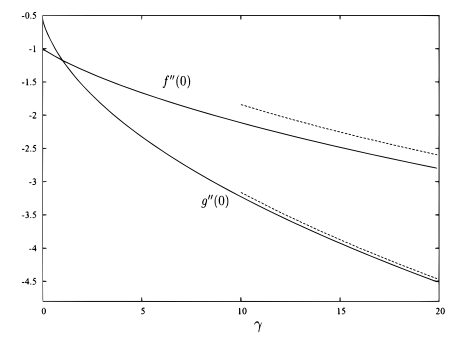

Figure 2. Impermeable surface, $S=0$ : plots of $f^{\prime \prime}(0)$ and $g^{\prime \prime}(0)$ against $\gamma$ obtained from the numerical solution to equations (2.4)-(2.6). Asymptotic expressions (3.6) are shown by broken lines.

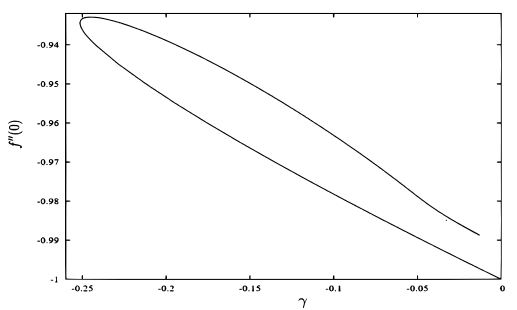

a)

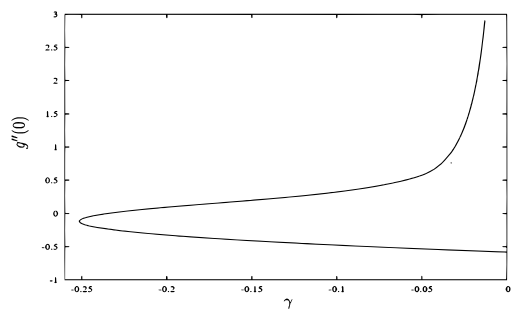

b)

Figure 3. Impermeable surface, $S=0$ : plots of a) $f^{\prime \prime}(0)$ and b) $g^{\prime \prime}(0)$ against $\gamma$ obtained from the numerical solution to equations $(2.4)-(2.6),(2.8)$.

whereas there is a much greater range over which $g^{\prime}$ changes, growing larger as $\gamma$ is further reduced. The values of $\eta$ where $g^{\prime}$ achieved its outer value are much greater than those used to plot this figure, a value of $\eta_{\infty}=50.0$ being used in the numerical integration.

Further insights into the nature of the solution for an impermeable surface can be gained by examining how the solution behaves for $\gamma$ large.

\subsection{Solution for $\gamma$ large}

To obtain a solution valid for $\gamma$ large we put

$$
f=\gamma^{-1 / 2} F, g=\gamma^{-1 / 2} G, \zeta=\gamma^{1 / 2} \eta .
$$

Equations (2.4)-(2.5) become

$$
\begin{aligned}
& F^{\prime \prime \prime}+G F^{\prime \prime}+\gamma^{-1}\left(F F^{\prime \prime}-F^{2}\right)=0, \\
& G^{\prime \prime \prime}+G G^{\prime \prime}-G^{2}+\gamma^{-1} F G^{\prime \prime}=0,
\end{aligned}
$$

subject to

$$
\begin{aligned}
& F(0)=0, \quad G(0)=0, \quad F^{\prime}(0)=1, \quad G^{\prime}(0)=1, \\
& F^{\prime} \rightarrow 0, \quad G^{\prime} \rightarrow 0 \text { as } \zeta \rightarrow \infty,
\end{aligned}
$$




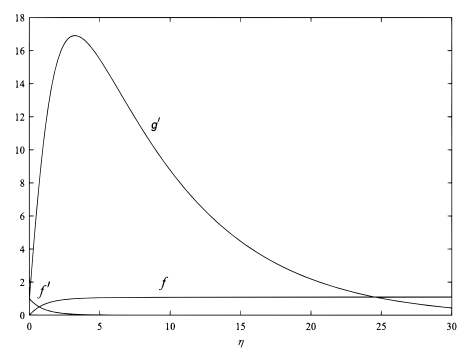

Figure 4. Impermeable surface, $S=0$ : profile plots of $f, f^{\prime}$ and $g^{\prime}$ against $\eta$ for the upper branch solution with $\gamma=-0.01253$ obtained from the numerical solution to equations (2.4)-(2.6), (2.8).

where primes now denote differentiation with respect to $\zeta$. The leading-order problem for an impermeable wall is

$$
\begin{aligned}
& F_{0}^{\prime \prime \prime}+G_{0} F_{0}^{\prime \prime}=0, G_{0}^{\prime \prime \prime}+G_{0} G_{0}^{\prime \prime}-G_{0}^{2}=0, \\
& F_{0}(0)=0, \quad G_{0}(0)=0, \quad F_{0}^{\prime}(0)=1, \\
& G_{0}^{\prime}(0)=1, \quad F_{0}^{\prime} \rightarrow 0, \quad G_{0}^{\prime} \rightarrow 0 \text { as } \zeta \rightarrow \infty .
\end{aligned}
$$

Equations (3.2)-(3.3) have the solution

$$
G_{0}=1-e^{-\zeta}, F_{0}^{\prime}=C_{0} \exp \left(e^{-\zeta}\right)+D_{0}
$$

where the constants $C_{0}$ and $D_{0}$ are determined from the boundary conditions as

$$
C_{0} e^{-1}+D_{0}=1, C_{0}+D_{0}=0
$$

giving

$$
C_{0}=-D_{0}=-e /(e-1) \text { and } F_{0}^{\prime \prime}(0)=-1 /(e-1) .
$$

From (3.1), (3.4), (3.5) we then have

$$
f^{\prime \prime}(0) \sim-0.58198 \gamma^{1 / 2}+\ldots, g^{\prime \prime}(0) \sim-\gamma^{1 / 2}+\ldots \text { as } \gamma \rightarrow \infty .
$$

Asymptotic expressions (3.6) are also shown in Figure 2 by broken lines and show good agreement with the numerical values, though with $g^{\prime \prime}(0)$ showing better agreement than $f^{\prime \prime}(0)$.

\section{Permeable surface, $S \neq 0$}

Here we solve equations (2.4)-(2.5) subject to boundary conditions (2.6), (2.8). In Figure 5 we plot $f^{\prime \prime}(0)$ against $S$ for $\gamma=1$, noting that for this value of $\gamma, g^{\prime \prime}(0)=f^{\prime \prime}(0)$. We see that the solution continues to large positive $S$, with $f^{\prime \prime}(0)$ negative and $\left|f^{\prime \prime}(0)\right|$ increasing, and to large negative $S$ with $f^{\prime \prime}(0)$ negative and increasing towards zero as $|S|$ is increased. 


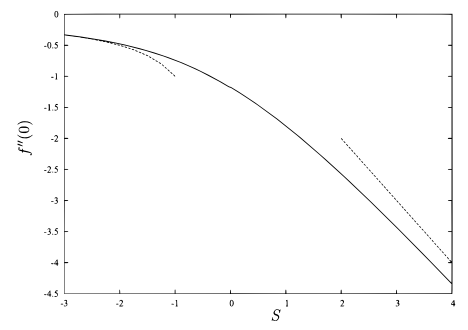

Figure 5. Permeable surface, $S \neq 0$ : plot of $f^{\prime \prime}(0)$ against $S$ for $\gamma=1$ obtained from the numerical solution to equations (2.4)-(2.6), (2.8). Asymptotic expressions (4.7), for $S$ large, and (4.26), for $|S|$ large $S<0$, are shown by broken lines. For this value of $\gamma$, $g^{\prime \prime}(0)=f^{\prime \prime}(0)$.

In Figure 6 we plot $f^{\prime \prime}(0)$ and $g^{\prime \prime}(0)$ against $S$ for $\gamma=-1$. Here, there is a critical value $S_{c}$ of $S$ with $S_{c} \simeq 1.4487$ limiting the range of possible solutions. The solution again continues to large positive $S$ with both $f^{\prime \prime}(0)$ and $g^{\prime \prime}(0)$ negative with, on the lower solution branch, $\left|f^{\prime \prime}(0)\right|$ and $\left|g^{\prime \prime}(0)\right|$ increasing as $S$ is increased. On the upper solution branch, both $f^{\prime \prime}(0)$ and $g^{\prime \prime}(0)$ again continue to large positive $S$ but now the values of $\left|g^{\prime \prime}(0)\right|$ decrease as $S$ is increased.

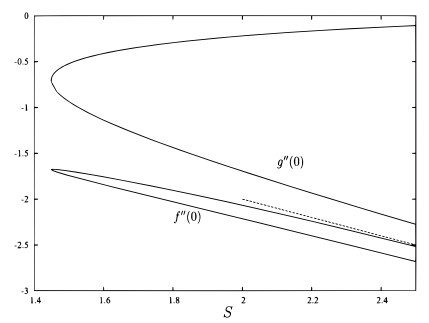

Figure 6. Permeable surface, $S \neq 0$ : plots of $f^{\prime \prime}(0)$ and $g^{\prime \prime}(0)$ against $S$ for $\gamma=-1$ obtained from the numerical solution to equations (2.4)-(2.6), (2.8). Asymptotic expression (4.7), for $S$ large, is shown by the broken line.

The variation of the solution with $\gamma$ is shown in Figure 7 where we plot $f^{\prime \prime}(0)$ and $g^{\prime \prime}(0)$ against $\gamma$ for $S=1.0$. There is a critical value at $\gamma=\gamma_{c} \simeq-0.6838$, with two solution branches in $\gamma>\gamma_{c}$. One branch passes through the solution for $\gamma=0$ given by $(2.9)-(2.10)$ with $f^{\prime \prime}(0)=-1.6180, g^{\prime \prime}(0)=-1.3286$ and continues to large positive $\gamma$. On the other solution branch, $f^{\prime \prime}(0)$ appears to loop back to the value given above for $\gamma=0$. However, a singularity appears in the solution for $g$ with $g^{\prime \prime}(0)$ becoming large and positive as $\gamma \rightarrow 0$ from below. Similar behaviour was seen for the other values of $S$ tried, both positive and negative. 


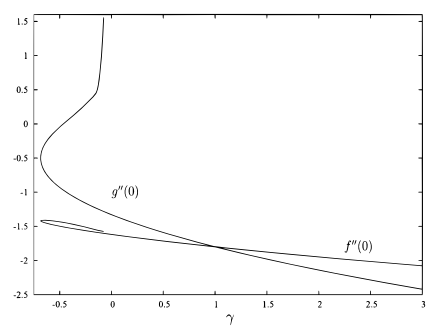

Figure 7. Permeable surface, $S \neq 0$ : plots of $f^{\prime \prime}(0)$ and $g^{\prime \prime}(0)$ against $\gamma$ for $S=1$ obtained from the numerical solution to equations $(2.4)-(2.6),(2.8)$.

\subsection{Solution for $\gamma$ large}

As seen in Figure 7 one solution branch continues to large $\gamma$. To obtain a solution valid in this limit we start by noting that our previous discussion requires $S$ to be small, of $O\left(\gamma^{-1 / 2}\right)$, and in this case the limiting forms for $f^{\prime \prime}(0)$ and $g^{\prime \prime}(0)$ are given by (3.6) to leading order. To obtain a solution when $S$ is of $O(1)$ we put

$$
f=\bar{F}(\bar{\zeta}), \quad g=\gamma^{-1 / 2} \bar{G}, \quad \bar{\zeta}=\gamma^{1 / 2} \eta .
$$

Equations (2.4)-(2.6) give

$$
\begin{aligned}
& \bar{F}^{\prime \prime \prime}+\bar{G} \bar{F}^{\prime \prime}+\gamma^{-1 / 2}\left(\bar{F} \bar{F}^{\prime \prime}-\bar{F}^{2}\right)=0 \\
& \bar{G}^{\prime \prime \prime}+\bar{G} \bar{G}^{\prime \prime}-\bar{G}^{2}+\gamma^{-1 / 2} \bar{F} \bar{G}^{\prime \prime}=0
\end{aligned}
$$

subject to

$$
\begin{gathered}
\bar{F}(0)=S, \bar{G}(0)=0, \bar{F}^{\prime}(0)=\gamma^{-1 / 2}, \\
\bar{G}^{\prime}(0)=1, \bar{F}^{\prime} \rightarrow 0, \bar{G}^{\prime} \rightarrow 0 \text { as } \bar{\zeta} \rightarrow \infty,
\end{gathered}
$$

where primes now denote differentiation with respect to $\bar{\zeta}$. We look for a solution by expanding $\bar{F}=\bar{F}_{0}+\gamma^{-1 / 2} \bar{F}_{1}+\ldots, \bar{G}=\bar{G}_{0}+\gamma^{-1 / 2} \bar{G}_{1}+\ldots$ At leading order we find $\bar{F}_{0} \equiv S$ and, following $(3.4), \bar{G}_{0}=1-e^{-\bar{\zeta}}$.

At $O\left(\gamma^{-1 / 2}\right)$ we then have

$$
\bar{F}_{1}^{\prime \prime \prime}+\left(1-e^{-\bar{\zeta}}\right) \bar{F}_{1}^{\prime \prime}=0, \bar{F}_{1}(0)=0, \bar{F}_{1}^{\prime}(0)=0, \bar{F}_{1}^{\prime} \rightarrow 0 \text { as } \bar{\zeta} \rightarrow \infty .
$$

Again following (3.4)-(3.5) we have

$$
\bar{F}_{1}^{\prime}=\frac{e}{(e-1)}\left(1-\exp \left(-e^{-\bar{\zeta}}\right)\right.
$$

This gives

$$
f^{\prime \prime}(0) \sim-0.58198 \gamma^{1 / 2}+\ldots, g^{\prime \prime}(0) \sim-\gamma^{1 / 2}+\ldots \text { as } \gamma \rightarrow \infty .
$$

It is not clear from Figure 7 that these limits are being approached. However, our numerical integrations taken to very much larger values of $\gamma$ do indicate that this is the case. Also these integrations show a thinning of the flow region as $\gamma$ is increased, consistent with transformation (4.1). 


\subsection{Critical values}

From Figure 3 we see that, for $S=0$, there is a critical value $\gamma_{c}$ of $\gamma$ and that, from Figures 6 and 7, this critical value depends on $S$. We calculate how the critical values $\gamma_{c}$ vary with $S$ using the method described in [22,23], for example, with $\gamma_{c}$ being plotted against $S$ in Figure 8. We see that $\gamma_{c}<0$ throughout and that $\gamma_{c}$ becomes large and negative as $S$ is increased. The values of $\gamma_{c}$ become smaller, approaching zero, as $S$ is decreased in the negative direction, suggesting that, for large negative values of $S$, there will be a solution only for $\gamma \geq 0$

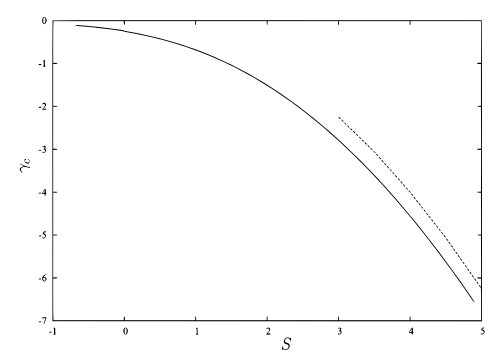

Figure 8. Plot of the critical values $\gamma_{c}$ against $S$, asymptotic expression (4.12) is shown by the broken line.

\subsection{Solution for $S$ large $(S>0$, strong suction $)$ - lower solution branch}

We start by assuming that $\gamma$ is of $O(1)$ and put

$$
f=S+S^{-1} \phi, g=S^{-1} h, \xi=S \eta .
$$

Equations (2.4)-(2.5) become

$$
\begin{aligned}
\phi^{\prime \prime \prime}+\phi^{\prime \prime}+S^{-2}\left(\phi \phi^{\prime \prime}-\phi^{2}+\gamma h \phi^{\prime \prime}\right) & =0, \\
h^{\prime \prime \prime}+h^{\prime \prime}+S^{-2}\left(\gamma h h^{\prime \prime}-\gamma h^{\prime 2}+\phi h^{\prime \prime}\right) & =0,
\end{aligned}
$$

subject to the boundary conditions

$$
\phi(0)=0, h(0)=0, \phi^{\prime}(0)=1, h^{\prime}(0)=1, \phi^{\prime}, h^{\prime} \rightarrow 0 \text { as } \xi \rightarrow \infty,
$$

where primes now denote differentiation with respect to $\xi$. The leading-order problem for $S$ large has the solution

$$
\phi=1-e^{-\xi}, h=1-e^{-\xi},
$$

giving

$$
f^{\prime \prime}(0) \sim-S+\ldots, g^{\prime \prime}(0) \sim-S+\ldots, \text { as } S \rightarrow \infty .
$$

Asymptotic expression (4.7) is shown in Figures 5 and 6 by broken lines showing good agreement with the numerically determined values even at relatively small 
values of $S$ for the upper branch solutions, improving as $S$ is increased further and better for $f^{\prime \prime}(0)$ than for $g^{\prime \prime}(0)$ in Figure 6 .

When $\gamma$ is large, particularly from equations (4.3)-(4.4) when $\gamma$ is of $O\left(S^{2}\right)$, the above solution does not hold. To deal with this case we put $\gamma=\mu S^{2}$, with $\mu$ of $O(1)$ and still apply transformation (4.2). At leading order for $S$ large, we now have

$$
\phi^{\prime \prime \prime}+\phi^{\prime \prime}+\mu h \phi^{\prime \prime}=0, \quad h^{\prime \prime \prime}+\mu\left(h h^{\prime \prime}-h^{2}\right)+h^{\prime \prime}=0,
$$

still subject to boundary conditions (4.5). When $\mu=0$, we recover expressions (4.6). When $\mu>0$, equation (4.8) has a solution of the form

$$
h=\frac{1}{a \mu^{1 / 2}}\left(1-e^{-a \mu^{1 / 2} \xi}\right) \text { for some } a=a(\mu)>0 .
$$

Substituting (4.9) into equation (4.8) we obtain, for $\mu>0$,

$$
\mu a^{2}-\mu^{1 / 2} a-\mu=0 \text { giving } a=(1+\sqrt{1+4 \mu}) /\left(2 \mu^{1 / 2}\right) .
$$

Hence, for $\mu>0$, there is only the one solution which gives

$$
g^{\prime \prime}(0) \sim-\frac{1+\sqrt{1+4 \mu}}{2} S+\ldots \text { as } S \rightarrow \infty .
$$

When $\mu<0$ we get

$$
h=\frac{1}{a|\mu|^{1 / 2}}\left(1-e^{-a|\mu|^{1 / 2} \xi}\right) .
$$

Equation (4.8) now gives

$$
|\mu| a^{2}-|\mu|^{1 / 2} a+|\mu|=0 \text { with } a=\frac{1 \pm \sqrt{1-4|\mu|}}{2|\mu|^{1 / 2}} .
$$

Expression (4.10) requires $|\mu| \leq 1 / 4$ or $0>\mu>-1 / 4$ and, for $\mu>-1 / 4$, there are two solutions which have

$$
g^{\prime \prime}(0) \sim-\frac{1+\sqrt{1+4 \mu}}{2} S+\ldots, \quad g^{\prime \prime}(0) \sim-\frac{1-\sqrt{1+4 \mu}}{2} S+\ldots \text { as } S \rightarrow \infty
$$

and a critical point at $\mu=-1 / 4$ giving

$$
\gamma_{c} \sim-\frac{S^{2}}{4}+\ldots \text { as } S \rightarrow \infty
$$

Asymptotic expression (4.12) is also shown in Figure 8 by a broken line. There appears to be good agreement between this expression and the the values obtained numerically even at quite moderate values of $S$. There also seems to be an $O(1)$ difference between the numerical values and asymptotic expression (4.12) consistent with the scaling (4.2). Expressions (4.11) give

$$
g^{\prime \prime}(0) \sim-\left(S+\gamma S^{-1}+\ldots\right), g^{\prime \prime}(0) \sim \gamma S^{-1}+\ldots,
$$

for the lower and upper branch solutions respectively. 


\subsection{Solution for $S$ large $(S>0$, strong suction) - upper solution branch}

We see from Figure 6 that, for $\gamma<0$, there are two solution branches each proceeding to large positive $S$ with the solution for $f^{\prime \prime}(0)$ approaching the limit described above. However, the behaviour of $g^{\prime \prime}(0)$ for large $S$ is different on the upper branch and to describe this solution, assuming that $\gamma<0$, we start in an inner region where we again make transformation (4.2). The solution for $\phi$ proceeds as above with the leading-order term given in (4.6). We now expand $h$ as

$$
h(\xi)=h_{0}(\xi)+S^{-2} h_{1}(\xi)+S^{-4} h_{2}(\xi)+\ldots
$$

and take $h_{0}=\xi$. At $O\left(S^{-2}\right)$ we then have

$$
h_{1}^{\prime \prime \prime}+h_{1}^{\prime \prime}=-\phi_{0} h_{1}^{\prime \prime}+\gamma\left(h_{0}^{\prime 2}-h_{0} h_{0}^{\prime \prime}\right)=\gamma, h_{1}(0)=h_{1}^{\prime}(0)=0,
$$

giving $h_{1}=\gamma \xi^{2} / 2$. A further calculation gives

$$
h_{2}=\frac{\gamma^{2} \xi^{3}}{6}-\frac{\left(\gamma^{2}+\gamma\right) \xi^{2}}{2}+\gamma(1+\xi) e^{-\xi}-\gamma
$$

We now consider an outer region where we take $f=S+O\left(S^{-1}\right)+\ldots$ (the outer form for $f$ in the inner region) and put

$$
g=S H, \quad y=S^{-1} \eta,
$$

with equation (2.5) giving

$$
(1+\gamma H) H^{\prime \prime}-\gamma H^{\prime 2}=-S^{-2}\left(H^{\prime \prime \prime}+H^{\prime \prime}\right), H^{\prime} \rightarrow 0 \text { as } y \rightarrow \infty,
$$

where primes now denote differentiation with respect to $y$. Matching with the inner region gives

$$
H \sim y+\frac{\gamma y^{2}}{2}+\frac{\gamma^{2} y^{3}}{6}+\ldots+S^{-2}\left(-\frac{\left(\gamma^{2}+\gamma\right) y^{2}}{2}+\ldots\right)+O\left(S^{-4}\right) .
$$

An expansion of the form $H(y ; S)=H_{0}(y)+S^{-2} H_{1}(y)+\ldots$ is suggested by (4.17)-(4.18). At leading order we have

$$
\left(1+\gamma H_{0}\right) H_{0}^{\prime \prime}-\gamma H_{0}^{\prime 2}=0 \quad \text { giving } \quad H_{0}=-\gamma^{-1}\left(1-e^{\gamma y}\right),
$$

on matching with the inner region and noting that $\gamma<0$. Applying (4.19) in the equation arising at $O\left(S^{-2}\right)$ then gives

$$
H_{1}^{\prime \prime}-2 \gamma H_{1}^{\prime}-\gamma^{2} H_{1}=-\left(\gamma^{2}+\gamma\right), H_{1}^{\prime} \rightarrow 0 \text { as } y \rightarrow \infty .
$$

Equation (4.20) has the solution, on matching with the inner region,

$$
H_{1}=(\gamma+1)\left((1-\gamma y) e^{\gamma y}-1\right) \text {. }
$$

From (4.14)-(4.16)

$$
g^{\prime \prime}(0) \sim S^{-1}\left(\gamma-\left(\gamma^{2}+2 \gamma\right) S^{-2}\right)+\ldots \text { as } S \rightarrow \infty
$$


consistent with the results shown in Figure 6, expressions (4.13) and our numerical integrations. The double-layer structure, whereby $f^{\prime}$ and $g^{\prime}$ vary over very different length scales and described above for the upper branch solutions for $S$ large, can be clearly seen in Figure 9 where we plot $f^{\prime}$ and $g^{\prime}$ against $\eta$ for $S=3.77$ and $\gamma=-1$. Again this numerical integration was taken to much larger values of $\eta$ than plotted in the figure to ensure that the outer boundary condition on $g^{\prime}$ was fully satisfied.

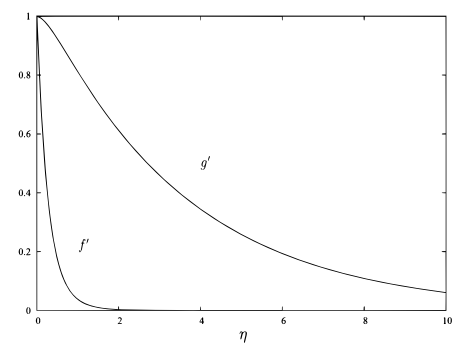

Figure 9. Permeable surface, $S \neq 0$ : profile plots of $f^{\prime}$ and $g^{\prime}$ against $\eta$ for the upper branch solution for $S=3.77$ with $\gamma=-1.0$ obtained from the numerical solution to equations (2.4)-(2.6), (2.8).

\subsection{Solution for $|S|$ large $(S<0$, strong blowing)}

To obtain a solution valid for $|S|$ large, $S<0$, we start in an inner region where we write, assuming that $\gamma>0$,

$$
f=|S| U, \quad g=|S| V, \quad Y=\eta|S|^{-1} .
$$

Transformation (4.22) gives, at leading order,

$$
U U^{\prime \prime}-U^{\prime 2}+\gamma V U^{\prime \prime}=0, \quad \gamma\left(V V^{\prime \prime}-V^{\prime 2}\right)+U V^{\prime \prime}=0,
$$

subject to

$$
U(0)=-1, \quad V(0)=0, \quad U^{\prime}(0)=1, \quad V^{\prime}(0)=1,
$$

the outer boundary conditions are relaxed at this stage and where primes now denote differentiation with respect to $Y$. From (4.23)-(4.24) we have

$$
U^{\prime \prime}=\frac{U^{\prime 2}}{U+\gamma V}, \quad V^{\prime \prime}=\frac{\gamma V^{\prime 2}}{U+\gamma V},
$$

giving

$$
U^{\prime \prime}(0)=-1, \quad V^{\prime \prime}(0)=-\gamma,
$$

so that

$$
f^{\prime \prime}(0) \sim S^{-1}+\ldots, g^{\prime \prime}(0) \sim \gamma S^{-1}+\ldots \text { as } S \rightarrow-\infty .
$$

Asymptotic expression (4.26) is shown in Figure 5, also by a broken line, showing very good agreement with the numerical values even at relatively small values of $|S|$. 
An outer region is required to satisfy the outer boundary conditions and this is, perhaps, most easily described for the case when $\gamma=1$. In this case

$$
U=-1+Y-\frac{Y^{2}}{2}, V=Y-\frac{Y^{2}}{2}
$$

giving

$$
U^{\prime}=V^{\prime}=0 \text { at } Y=1, \eta=|S| .
$$

Expression (4.27) suggests that, for the outer region, we put $\eta=\bar{\eta}+|S|$ and then

$$
f=-\frac{|S|}{2}+|S|^{-1 / 3} \bar{f}, g=\frac{|S|}{2}+|S|^{-1 / 3} \bar{g}, \bar{\eta}=|S|^{-1 / 3} y
$$

For this case we find $\bar{g} \equiv \bar{f}$ and then

$$
\bar{f}^{\prime \prime \prime}+2 \bar{f} \bar{f}^{\prime \prime}-\bar{f}^{2}=0
$$

where primes now denote differentiation with respect to $\bar{\eta}$ and subject to, on matching with the inner solution (4.27),

$$
\bar{f} \sim-\frac{\bar{\eta}^{2}}{2}+\ldots \text { as } \bar{\eta} \rightarrow-\infty, \bar{f}^{\prime} \rightarrow 0 \text { as } \bar{\eta} \rightarrow \infty .
$$

When $\gamma=0$, equation (4.25) gives $U=-e^{-Y}$ which can also obtained from (2.9) in the limit as $S \rightarrow-\infty$. This gives the same asymptotic expression for $f^{\prime \prime}(0)$ in (4.26). From expression (2.9), $c \sim|S|^{-1}+\ldots$ for $|S|$ large. This leads to

$$
g^{\prime} \sim 1+e^{-1}|S|^{2}-\exp \left(-|S|^{2} e^{-\eta /|S|}\right)+\ldots,
$$

giving

$$
g^{\prime \prime}(0) \sim-|S| e^{-|S|^{2}}+\ldots,
$$

as $S \rightarrow-\infty$. Expression (4.28) suggests that the solution for $|S|$ large cannot be continued into $\gamma<0$ indicating that a solution can proceed to large $S$ only if $\gamma \geq 0$, as is also borne out by our numerical solutions.

\subsection{Temporal stability}

To determine the temporal stability of the solutions when multiple solutions exist, as seen in Figures 3, 6 and 7 we construct an initial-value problem, as was done previously in Merkin [21] and more recently in Lok et al. [17], with the steady states of this initial-value problem being given by equations (2.4), (2.5). The stability is then determined by looking for a solution in the form

$$
f(\eta, t)=f_{0}(\eta)+e^{\lambda t} f_{1}(\eta), \quad g(\eta, t)=g_{0}(\eta)+e^{\lambda t} g_{1}(\eta)
$$

where $f_{0}, g_{0}$ are now the steady state solution. When expressions (4.29) are substituted into the corresponding initial-value problem we obtain a linear problem for the eigenvalue $\lambda$, namely

$$
\begin{aligned}
& f_{1}^{\prime \prime \prime}+f_{0} f_{1}^{\prime \prime}-2 f_{0}^{\prime} f_{1}^{\prime}+f_{0}^{\prime \prime} f_{1}+\gamma\left(g_{0} f_{1}^{\prime \prime}+f_{0}^{\prime \prime} g_{1}\right)-\lambda f_{1}^{\prime}=0, \\
& g_{1}^{\prime \prime \prime}+\gamma\left(g_{0} g_{1}^{\prime \prime}-2 g_{0}^{\prime} g_{1}^{\prime}+g_{0}^{\prime \prime} g_{1}\right)+g_{0}^{\prime \prime} f_{1}+f_{0} g_{1}^{\prime \prime}-\lambda g_{1}^{\prime}=0
\end{aligned}
$$


Table 2. The smallest eigenvalue $\lambda$ for several values of $\gamma<0$ when $S=1$.

\begin{tabular}{ccc}
\hline$\gamma$ & $\lambda$ & $\lambda$ \\
\hline & upper branch & lower branch \\
\hline-0.68 & 0.064471 & -0.066169 \\
-0.65 & 0.181322 & -0.196054 \\
-0.60 & 0.263845 & -0.299141 \\
-0.50 & 0.332414 & -0.409860 \\
-0.40 & 0.341931 & -0.479353 \\
\hline
\end{tabular}

subject to homogeneous boundary conditions and the extra condition that $f_{1}^{\prime \prime}(0)=1$ to force a nontrivial solution. The value of $\lambda$ obtained from the numerical solution of (4.30) then determines the stability, with the solution being stable if $\lambda<0$ and unstable if $\lambda>0$.

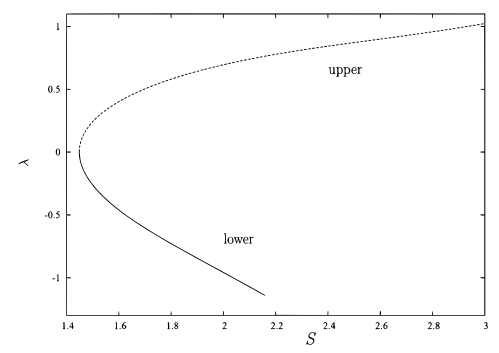

Figure 10. Temporal stability: a plot of the eigenvalue $\lambda$ against $S$ for $\gamma=-1.0$, solution stable where $\lambda<0$ (full line), unstable where $\lambda>0$ (broken line).

We illustrate this in Figure 10 with a plot of $\lambda$ against $S$ for the case shown in Figure 6 when $\gamma=-1.0$. This Figure shows that the lower branch is stable and that the upper branch is unstable. The change in stability occurs at the critical point $S_{c} \simeq-1.4487$ where $\lambda=0$, i.e. at the saddle-node bifurcation at $S_{c}$. In Table 2 we give the values of $\lambda$ for representative values of $\gamma$ when $S=1$, the case shown in Figure 7 showing that the upper branch is unstable and the lower branch is stable. Here the critical value is at $\gamma_{c} \simeq-0.6838$ and we see that the values of $\lambda$ become smaller close to this value of $\lambda$. When we consider the behaviour for $\gamma$ large, in effect applying transformation (3.1) in equations (4.30), we find that we require $\lambda$ to be of $O(\gamma)$ and, on writing $\mu=\gamma \lambda$, we find the the eigenvalue $\mu \simeq-0.45629$, independent of the parameter $S$, showing that these solutions are stable. From this we can deduce that any branch of solutions that proceeds to large $\gamma$ will be stable. The stability changes at the saddle-node bifurcation at the critical point where $\lambda=0$ with the second solution branch resulting from this bifurcation then being unstable. 


\section{Conclusions}

We have considered the non-symmetric flow over a stretching/shrinking surface in an otherwise quiescent fluid. We assumed that the surface could stretch or shrink in one direction and stretch in a direction perpendicular to this. We reduced the problem to similarity form by a suitable transformation of variables finding that the problem is described by two dimensionless parameters, $\gamma$ the relative stretching/shrinking rate and $S$ characterizing the fluid transfer through the boundary. We started by considering an impermeable surface, noting that there was a simple solution, (2.9)-(2.10) when $\gamma=0$. We found solutions for all cases when there is stretching in both directions, $\gamma>0$, Figure 2, with an asymptotic expression (3.6) for $\gamma$ large being obtained. However, when there is shrinking in one direction, $\gamma<0$, there is a critical value of $\gamma$ limiting the range of solution, Figure 3, i.e restricting the rate of shrinking and showing that dual solutions appear.

We then considered a permeable surface, Figure 5, when there is stretching in both directions and when there is shrinking in one direction, Figure 6 . In this latter case we again saw the existence of a critical value $\gamma_{c}$ of $\gamma$ with this now dependent on the surface fluid transfer rate $S$, Figure 6 and 7 , with $\gamma_{c}$ being plotted in Figure 8. These critical values led to two solution branches both proceeding to large values of $S$, strong withdrawal rates, and asymptotic expressions (4.13) and (4.21) were obtained for $S$ large for both solution branches. This latter case exhibited a double-region structure in which the length scales over which $f^{\prime}$ and $g^{\prime}$ varied became increasingly different as the values of $S$ are increased. The main effect of fluid transfer through the wall, when there is shrinking in one direction, is to increase the range of possible solutions when there is fluid withdrawal, increasingly more so as the rate of fluid withdrawal is increased. However, fluid injection through the wall decreases this range of possible solutions, limiting solutions only to stretching in both directions if this fluid injection is sufficiently strong.

\section{Acknowledgements}

The work of I. Pop has been supported from the grant PN-III-P4-ID-PCE-20160036, UEFISCDI, Romania. Y.Y. Lok would like to acknowledge the financial support received from the Ministry of Higher Education, Malaysia under the Fundamental Research Grant Scheme (203/PJJAUH/6711665).

\section{References}

[1] NAG: Numerical Algorithm Group. Available from Internet: https://www .nag. co.uk.

[2] M.E. Ali. On thermal boundary layer on a power-law stretched surface with suction or injection. Int. J. Heat Fluid Flow, 16(4):280-290, 1995. https://doi.org/10.1016/0142-727X(95)00001-7.

[3] P.D. Ariel. Generalized three-dimensional flow due to a stretching sheet. J. App. Math. Mech. (ZAMM), 83(12):844-852, 2003. https://doi.org/10.1002/zamm.200310052. 
[4] W.H.H. Banks. Similarity solutions of the boundary-layer equations for a stretching wall. J. Theor. Appl. Mech., 2:375-392, 1983.

[5] R.C. Bataller. Similarity solutions for flow and heat transfer of a quiescent fluid over a nonlinearly stretching surface. J. Mater. Processing Tech., 203(1-3):176183, 2008. https://doi.org/10.1016/j.jmatprotec.2007.09.055.

[6] C.K. Chen and M.I. Char. Heat transfer of a continuous, stretching surface with suction or blowing. J. Math. Anal. Appl., 135(2):568-580, 1988. https://doi.org/10.1016/0022-247X(88)90172-2.

[7] R. Cortell. Viscous flow and heat transfer over a nonlinearly stretching surface. Appl. Math. Computation, 184(2):864-873, 2007. https://doi.org/10.1016/j.amc.2006.06.077.

[8] L.J. Crane. Flow past a stretching plate. J. Appl. Math. Phys. (ZAMP), 21(4):645-647, 1970. https://doi.org/10.1007/BF01587695.

[9] T. Fang. Boundary layer flow over a shrinking sheet with powerlaw velocity. Int J. Heat Mass Transfer, 51(25-26):5838-5843, 2008. https://doi.org/10.1016/j.ijheatmasstransfer.2008.04.067.

[10] T.G. Fang, J. Zhang and S.S. Yao. Viscous flow over an unsteady shrinking sheet with mass transfer. Chin. Phys. Lett., 26:014703, 2009. https://doi.org/10.1088/0256-307X/26/1/014703.

[11] E.G. Fisher. Extrusion of Plastics. Wiley, New York, 1976.

[12] S. Goldstein. On backward boundary layers and flow in converging passages. J. Fluid Mech., 21(1):33-45, 1965. https://doi.org/10.1017/S0022112065000034.

[13] P.S. Gupta and A.S. Gupta. Heat and mass transfer on a stretching sheet with suction or blowing. Can. J. Chem. Eng., 55(6):744-746, 1977. https://doi.org/10.1002/cjce.5450550619.

[14] A. Ishak, R. Nazar and I. Pop. Hydromagnetic flow and heat transfer adjacent to a stretching vertical sheet. Heat Mass Transfer, 44:921-927, 2008. https://doi.org/10.1007/s00231-007-0322-z.

[15] S.J. Liao. A new branch of solutions of boundary-layer flows over an impermeable stretched plate. Int. J. Heat and Mass Transfer, 48(12):2529-2539, 2005. https://doi.org/10.1016/j.ijheatmasstransfer.2005.01.005.

[16] S.J. Liao and I. Pop. Explicit analytic solution for similarity boundary layer equations. Int. J. Heat Mass Transfer, 47(1):75-85, 2004. https://doi.org/10.1016/S0017-9310(03)00405-8.

[17] Y.Y. Lok, J.H. Merkin and I. Pop. Axisymmetric rotational stagnation point flow impinging on a permeable stretching/shrinking rotating disk. Eur. J. Mech. B/Fluids, 72(November-December):275-292, 2018. https://doi.org/10.1016/j.euromechflu.2018.05.013.

[18] E. Magyari and B. Keller. Exact solutions for self-similar boundary-layer flows induced by permeable stretching walls. Eur. J. Mech. B/Fluids, 19(1):109-122, 2000. https://doi.org/10.1016/S0997-7546(00)00104-7.

[19] E. Magyari and P.Weidman. New solutions of the Navier-Stokes equations associated with flow above moving boundaries. Acta Mech., 228(10):3725-3733, 2017. https://doi.org/10.1007/s00707-017-1919-z.

[20] J.B. McLeod and K.R. Rajagopal. On the uniqueness of flow of a Navier-Stokes fluid due to a stretching boundary. Arch. Ration. Mech. Anal., 98:385-393, 1987. https://doi.org/10.1007/BF00276915. 
[21] J.H. Merkin. On dual solutions occurring in mixed convection in a porous medium. J. Engng Math., 20:171-179, 1986. https://doi.org/10.1007/BF00042775.

[22] J.H. Merkin and T. Mahmood. Mixed convection boundary layer similarity solutions: prescribed wall heat flux. J. Applied Math. Physics (ZAMP), 40:5168, 1989. https://doi.org/10.1007/BF00945309.

[23] J.H. Merkin and I. Pop. Natural convection boundary-layer flow in a porous medium with temperature-dependent boundary conditions. Transp. Porous $\mathrm{Me}-$ dia, 85:397-414, 2010. https://doi.org/10.1007/s11242-010-9569-9.

[24] M. Miklavčič and C.Y. Wang. Viscous flow due to a shrinking sheet. Quart. Appl. Math., 64:283-290, 2006.

[25] M. Sajid, N. Ali, Z. Abbas and T. Javed. Stretching flows with general slip boundary condition. Int. J. Mod. Phys. B., 24(30):5939-5947, 2010. https://doi.org/10.1142/S0217979210055512.

[26] L.F. Shampine, I. Gladwell and S. Thompson. Solving ODEs with Matlab. Cambridge University Press, Cambridge, 2003.

[27] K. Vajravelu. Flow and heat transfer in a saturated porous medium over a stretching surface. J. App. Math. Mech. (ZAMM), 74(12):605-614, 1994. https://doi.org/10.1002/zamm.19940741209.

[28] C.Y. Wang. The three-dimensional flow due to a stretching flat surface. Phys. Fluids, 27(8):1915-1917, 1984. https://doi.org/10.1063/1.864868.

[29] C.Y. Wang. Review of similarity stretching exact solutions of the Navier-Stokes equations. Eur. J. Mech. B/Fluids, 30(5):475-479, 2011. https://doi.org/10.1016/j.euromechflu.2011.05.006.

[30] C.Y. Wang. Uniform flow over a bi-axial stretching surface. J. Fluids Engng., 137:084502, 2015. https://doi.org/10.1115/1.4029447. 